\title{
Diastolic function measurements and diagnostic consequences: a comparison of pulsed wave- and color-coded tissue Doppler imaging
}

\author{
Y. M. Hummel • I. J. T. Klip • R. M. de Jong • \\ P. G. Pieper • D. J. van Veldhuisen · A. A. Voors
}

Received: 8 October 2009/Accepted: 23 February 2010/Published online: 10 March 2010

(c) The Author(s) 2010. This article is published with open access at Springerlink.com

\begin{abstract}
Introduction Tissue Doppler imaging (TDI) plays an important role in assessing diastolic function using echocardiography. However, two different methods [pulsed wave (PW-TDI) and color-coded (CC-TDI)] are currently used. We aimed to compare both measurements.

Methods We included 114 patients that were referred to our echocardiography department for evaluation of diastolic left ventricular function. In these patients, we sequentially measured tissue velocities of basal lateral and septal myocardium of the left ventricle in an apical fourchamber view with both PW-TDI and CC-TDI.

Results Our cohort consisted of a heterogeneous group of patients with and without a history of cardiac disease. Mean age of the patients was $52 \pm 16.7$ years, and $62 \%$ were males. We found a strong correlation between PW-TDI- and CC-TDI-derived myocardial velocities $\left(r=0.93 ; p=0.001\right.$ ). However, $E^{\prime}$ (mean of lateral and septal) velocities measured with PW-TDI were consistently higher compared to CC-TDI values [PW-TDI $E^{\prime} 10.3 \pm$ 3.9 (SD) $\mathrm{cm} / \mathrm{s}$ vs. CC-TDI $\left.E^{\prime} 7.7 \pm 3.1 \mathrm{~cm} / \mathrm{s} ; p<0.001\right]$. From these data, we calculated that the relation between $E^{\prime}$ measured with PW-TDI and CC-TDI can be described as: $E^{\prime}(\mathrm{PW}-\mathrm{TDI})=1.25+1.17 \times E^{\prime}$ (CC-TDI). Consequently, $E / E^{\prime}$ measured with PW-TDI was consistently lower compared with CC-TDI $(9.1 \pm 3.1$ vs. $12.5 \pm 5.7$; $p<0.001)$ From these data, we calculated that the relation between $E / E^{\prime}$ measured with PW-TDI and CC-TDI can
\end{abstract}

Y. M. Hummel · I. J. T. Klip · R. M. de Jong ·

P. G. Pieper · D. J. van Veldhuisen · A. A. Voors $(\square)$ Department of Cardiology, University Medical Centre Groningen, University of Groningen, Hanzeplein 1, P.O. Box 30 001, 9700 RB Groningen, The Netherlands e-mail: a.a.voors@thorax.umcg.nl be described as: $E / E^{\prime}(\mathrm{PW}-\mathrm{TDI})=2.13+0.56 \times E / E^{\prime}$ (CC-TDI).

Conclusions Despite a strong correlation, tissue velocities measured with PW-TDI will yield higher values as compared with CC-TDI. This should be taken into account when defining cut-off values for the evaluation of diastolic function.

Keywords Tissue velocity - Diastolic function . Echocardiography · Tissue Doppler imaging

\section{Introduction}

Epidemiological studies have demonstrated that $38-54 \%$ of all patients, who are hospitalized for heart failure, have a preserved left ventricular ejection fraction (LVEF) [1,2]. In these patients, heart failure is considered to be caused by diastolic dysfunction. So far, large randomised clinical trials failed to demonstrate beneficial effects of treatments that are well validated in systolic heart failure [3, 4]. This might in part be caused by a lack of established parameters for the diagnosis of diastolic heart failure [5-7] which in many cases led to misdiagnosis of diastolic heart failure.

In a recent consensus statement of the European Society of Cardiology (ESC) [8] a diagnosis of diastolic heart failure should include signs and symptoms of heart failure, a relative preserved systolic heart function (LVEF $>50 \%$ ), echocardiographic evidence of diastolic dysfunction, and raised natriuretic peptides. $E / E^{\prime}$ was considered to be the most important diastolic function parameter, which is obtained through measuring mitral valve $E$ inflow velocity (early diastolic inflow) divided by myocardial $E^{\prime}$ velocity measured at the basal part of the lateral and septal LV wall. Several studies have shown that $E / E^{\prime}$ shows the best 
correlation with invasively measured pressure volume curves [9-12]. However, the actual value of $E / E^{\prime}$ depends on the method that has been used. $E^{\prime}$ values are derived from relatively new echo techniques which are generally accepted in the evaluation of diastolic LV function [13]. $E^{\prime}$ can be measured both by pulsed wave tissue Doppler imaging (PW-TDI) (spectral tissue Doppler imaging) and color-coded tissue Doppler imaging (CC-TDI). Both methods are based on the same tissue Doppler imaging (TDI) principle. However, differences between PW-TDI and CC-TDI measurements have been shown in phantoms and small cohorts of healthy volunteers and patients [14-16]. The primary aim of the present study was to assess similarities and differences between PW-TDI- and CC-TDI-derived $E^{\prime}$ values in a heterogeneous and larger group of patients referred for evaluation of diastolic function and to investigate the implications of using these different techniques for the classification of patients for diastolic heart failure using $E / E^{\prime}$.

\section{Methods}

Patient population

A total number of 114 consecutive patients, 18 years of age and older, who were referred for left ventricular diastolic function measurements at the Department of Echocardiography of the University Medical Center Groningen, were included in the present study. We excluded patients with a poor quality of the echocardiogram, atrial fibrillation/flutter or severe arrhythmias, hemodynamic important mitral valve regurgitation, patients with dyskinetic wall motions, and patients with artificial valves or congenital heart disease. The study was approved by the local medical ethics committee.

\section{Echocardiography}

Transthoracic echocardiography was performed using a Vivid-7 (General Electric, Horton, Norway) and a $2.5 \mathrm{MHz}$ probe. LV-dimension and wall thickness were measured on a standard parasternal long axis view, atrial dimensions measured in parasternal long axis and apical four-chamber view. Left ventricular systolic function was analyzed in all standard views. All measurements were performed according to the European Society of Echocardiography guidelines [17].

Standard mitral valve inflow parameters were measured in the apical four-chamber view with the sample volume at the tips of the mitral leaflets; two consecutive heart cycles were recorded at end-expiration. Early mitral valve inflow $(E)$, deceleration time of $E$ inflow (DT), atrial contraction
(A) and isovolumetric relaxation time (IVRT) were measured. Both TDI modalities were acquired. TDI measurements were performed in an apical four-chamber view $(\mathrm{AP} 4 \mathrm{CH})$ making sure samples were placed at the junction of MV annulus and basal part of LV myocardium. PW-TDI measurements were performed while scanning (online measurements) using a high-pass tissue filter. A sample size of $4 \mathrm{~mm}$ was used (standard setting of VIVID-7, GE, Horton, Norway). If needed, a sector tilt was used to make sure that the angle between the probe and myocardial motion was $<15 \%$. CC-TDI images were acquired and digitalized, exporting them to a workstation containing ECHOPAC 8.1 version software. 2D images with a high-pass tissue filter were digitalized, and CC-TDI measurements were performed offline, using a circular sample size of $3 \times 3 \mathrm{~mm}$ and FPS $>180$ frames/s, while a single observer was blinded by outcomes of PW-TDI measurements.

$E^{\prime}$ was measured with both TDI modalities by a single experienced echo technician. Mean of lateral and septal $E^{\prime}$ values were used in calculation of $E / E^{\prime}$.

\section{Statistical analysis}

Data were analyzed with SPSS version 14.0. All data were expressed as mean and standard deviation. CC-TDIand PW-TDI-derived values were compared using a paired $T$ test and Bland-Altman analysis. Scatter plots were made to evaluate linearity of $E^{\prime}$ and $E / E^{\prime}$ values. A simple regression analysis was used for calculating the relation between both methods of calculating $E^{\prime}$ and $E / E^{\prime}$. Agreement of classification was analyzed using Cohen's kappa test.

\section{Results}

A total of 114 patients were studied. Table 1 shows the patient characteristics. Mean age was $52 \pm 16.7$ years, and $62 \%$ were males. Nineteen patients had a previous myocardial infarction; chronic heart failure was diagnosed in 21 patients; 17 had a history of hypertension and 14 were diagnosed with coronary artery disease.

\section{Comparison between PW-TDI and CC-TDI}

We found a strong correlation of septal and lateral mean $E^{\prime}$ velocities between both PW-TDI and CC-TDI $(r=0.93$; $p=0.001$; Fig. 1). However, mean $E^{\prime}$ values measured with CC-TDI were consistently lower than those measured with PW-TDI. $E^{\prime}$ velocity measurements with PW-TDI showed a mean of $10.3 \pm 3.9 \mathrm{~cm} / \mathrm{s}$, and $E^{\prime}$ velocity measurements with CC-TDI show a mean of $7.7 \pm 3.1 \mathrm{~cm} / \mathrm{s}$ 
Table 1 Baseline characteristics of the patients

\begin{tabular}{|c|c|}
\hline Age (years) & $52 \pm 16.7$ \\
\hline Sex, male $(\%)$ & 62 \\
\hline $\mathrm{HF}(\mathrm{bpm})$ & $68 \pm 11.7$ \\
\hline Mean LVEF & $0.53 \pm 0.12$ \\
\hline \multicolumn{2}{|l|}{ LVEF category, $n(\%)$} \\
\hline $0.55-0.60$ & $90(78.9)$ \\
\hline $0.45-0.54$ & $8(7.1)$ \\
\hline $0.30-0.44$ & $11(9.6)$ \\
\hline$<0.30$ & $5(4.4)$ \\
\hline History of AMI, $n(\%)$ & $19(16.7)$ \\
\hline $\mathrm{CHF}, n(\%)$ & $21(18.4)$ \\
\hline \multicolumn{2}{|l|}{ NYHA, $n(\%)$} \\
\hline I & 93 (81.6) \\
\hline II & $11(9.6)$ \\
\hline III & $10(8.8)$ \\
\hline IV & $0(0)$ \\
\hline Hypertension, $n(\%)$ & $17(14.9)$ \\
\hline $\mathrm{CAD}, n(\%)$ & $14(12.3)$ \\
\hline$E$ & $0.84 \pm 0.23$ \\
\hline$A$ & $0.70 \pm 0.21$ \\
\hline$E / A$ ratio & $1.31 \pm 0.53$ \\
\hline DT & $224 \pm 79$ \\
\hline IVRT & $94 \pm 23$ \\
\hline $\mathrm{PW} E^{\prime}$ sep & $8.9 \pm 3.3$ \\
\hline $\mathrm{PW} E^{\prime}$ lat & $11.6 \pm 4.9$ \\
\hline Mean PW $E^{\prime}$ (sep-lat) & $10,3 \pm 3.9$ \\
\hline$E^{\prime}$ sep & $6.9 \pm 2.9$ \\
\hline$E^{\prime}$ lat & $8.5 \pm 3.7$ \\
\hline Mean $E^{\prime}$ (sep-lat) & $7.7 \pm 3.1$ \\
\hline$E / \mathrm{PW} E^{\prime}$ & $9.1 \pm 3.8$ \\
\hline$E / E^{\prime}$ & $12.5 \pm 5.7$ \\
\hline
\end{tabular}

Data are presented as mean $\pm \mathrm{SD}$

$P W E^{\prime} E$ myocardial velocity using pulsed wave tissue Doppler; $E^{\prime} E$ myocardial velocity using color-coded tissue Doppler; $E / P W E^{\prime} E / E^{\prime}$ calculated by usage of PW-TDI; $E / E^{\prime} E / E^{\prime}$ calculated by usage of CC-TDI; $H F$ heart frequency; $b p m$ beats per minute; $C H F$ chronic heart failure; $C A D$ coronary artery disease; NYHA New York Heart Association classification; $D T$ deceleration time; IVRT isovolumetric relaxation time; $L V E F$ left ventricular ejection fraction; $A M I$ acute myocardial infarction

$(p<0.001)$. Bland and Altman representations of these differences are shown in Fig. 2, showing a proportional error, i.e., the difference between both methodologies is higher when $E^{\prime}$ values are higher. The relation between $E^{\prime}$ measured in our data can be described as: $E^{\prime}$ (PW$\mathrm{TDI})=1.25+1.17 \times E^{\prime} \quad(\mathrm{CC}-\mathrm{TDI})$. Our Bland and Altman analysis (Fig. 3) shows the variability of the relationship between both measurements $(\sim 10-50 \%$ difference between values). We also found a strong correlation of $E / E^{\prime}$ values between PW-TDI and CC-TDI $(r=0.85$;

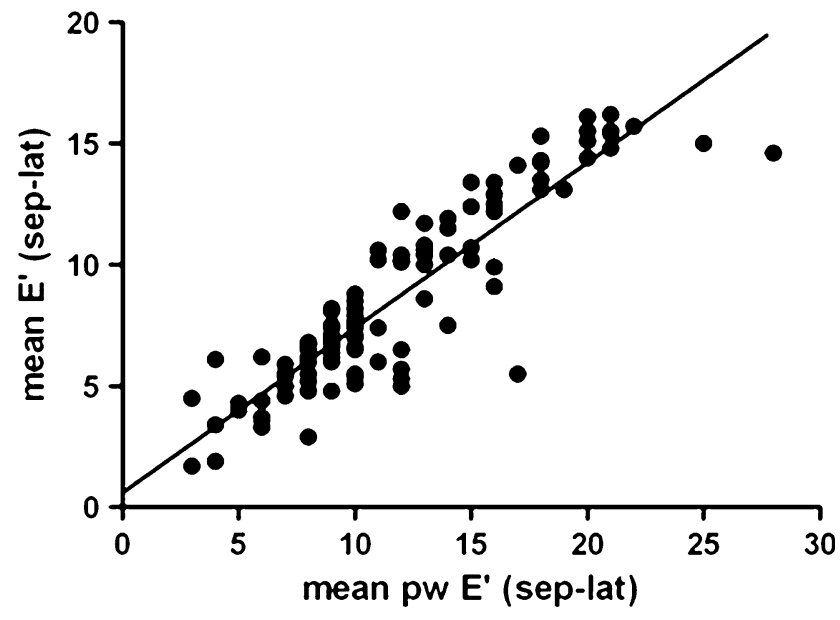

Fig. 1 Correlation between $E^{\prime}$ values measured with CC-TDI and PW-TDI $(r=0.93, p=0.001)$

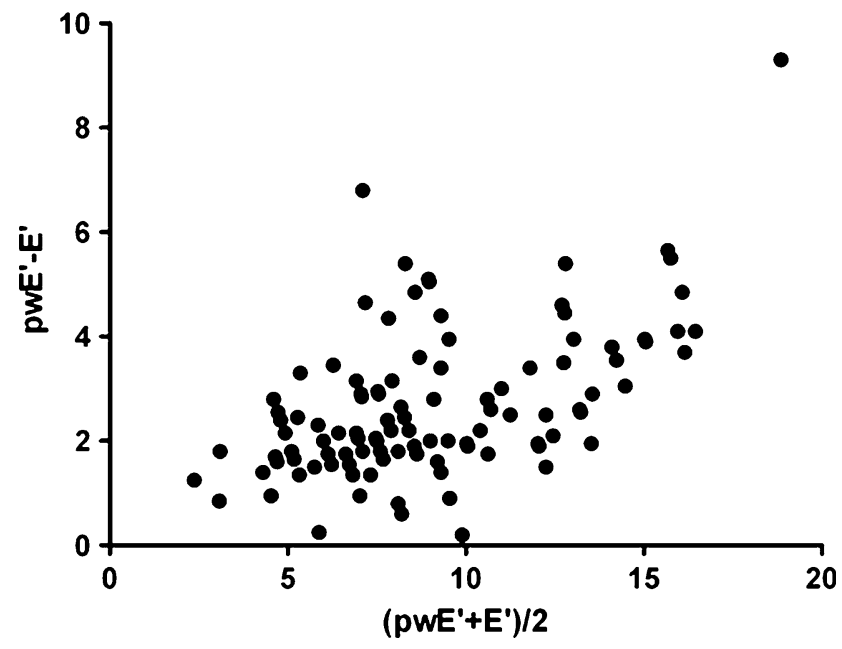

Fig. 2 Bland-Altman representation of $E^{\prime}$ values derived by PW-TDI and $\mathrm{CC}$-TDI

$p<0.001$; Fig. 4). However, E/E' measured with PW-TDI was consistently lower compared with CC-TDI $(9.1 \pm 3.1$ vs. $12.5 \pm 5.7 ; p<0.001)$. Relation between both methods of calculating $E / E^{\prime}$ can be formulated as: $E / E^{\prime}$ (PW-TDI) = $2.13+0.56 \times E / E^{\prime}(\mathrm{CC}-\mathrm{TDI})$

Classification of left ventricular diastolic function

The cut-off $E / E^{\prime}$ values for the diagnosis of diastolic heart failure defined by the ESC consensus statement (normal $<8$; possible diastolic dysfunction $8-15$; diastolic dysfunction $>15$ ) were based on measurements with PW-TDI. Using this definition, we found marked differences in the classification of diastolic function when using either PWTDI or CC-TDI. Diastolic dysfunction was diagnosed in nine patients using PW-TDI, and in 24 patients using CCTDI $(p<0.001)$. Conversely, absence of diastolic 


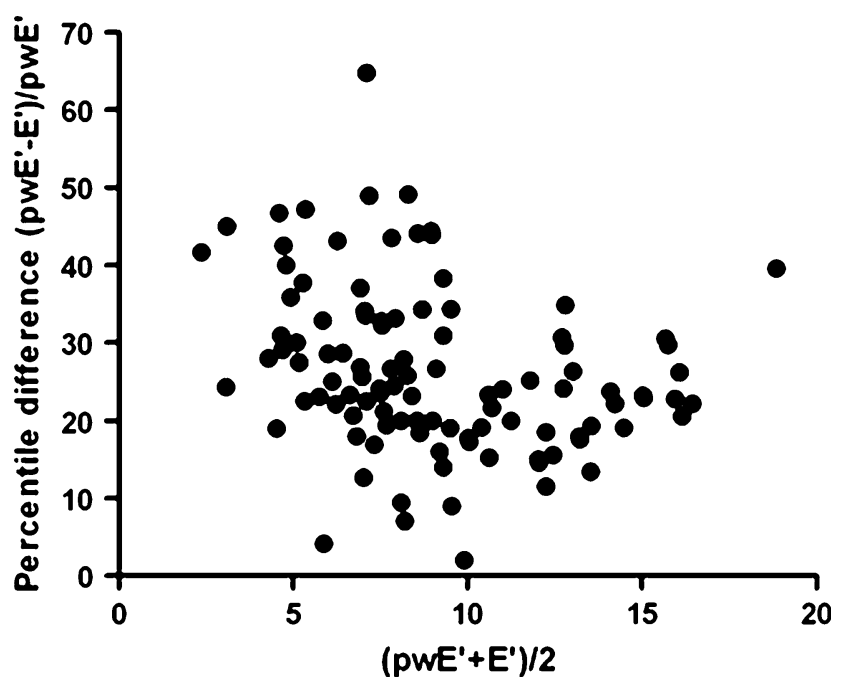

Fig. 3 Bland-Altman representation of $E^{\prime}$ values derived by PW-TDI and CC-TDI expressed in percentile differences

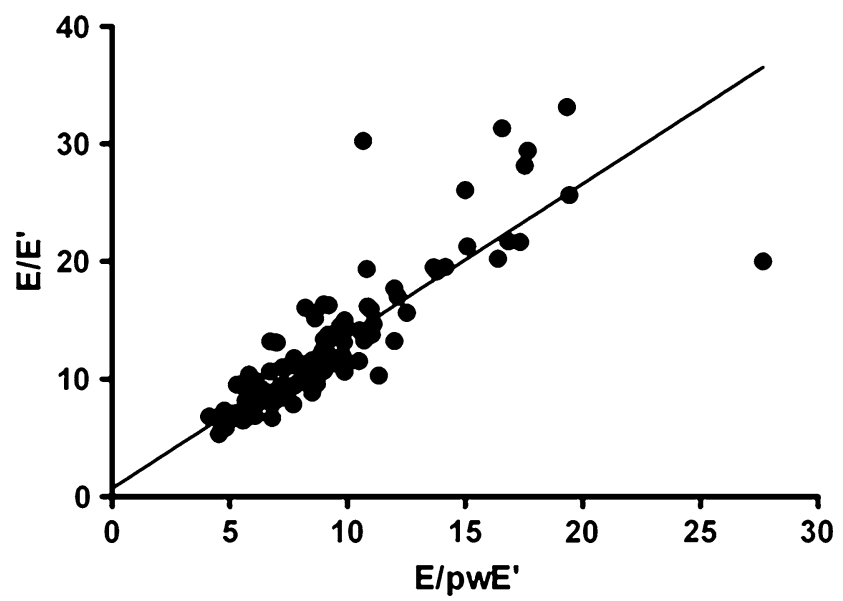

Fig. 4 Correlation between $E / E^{\prime}$ values measured with CC-TDI and PW-TDI $(r=0.85, p<0.001)$

dysfunction was diagnosed in 48 patients using PW-TDI, and in 16 patients using CC-TDI $(p<0.001)$ (Fig. 5). Using the proposed formula $E / E^{\prime}(\mathrm{PW}-\mathrm{TDI})=2.13+$ $0.56 \times E / E^{\prime}(\mathrm{CC}-\mathrm{TDI})$, cut-off values of CC-TDI should be: normal $<11$; possible diastolic dysfunction 11-20; diastolic dysfunction $>20$.

\section{Discussion}

We found a strong correlation between myocardial velocities measured with PW-TDI and CC-TDI in wide range of patients with and without left ventricular systolic and diastolic dysfunction and with and without symptoms. This indicates that although both methodologies are different, they represent the same velocity increase and decrease

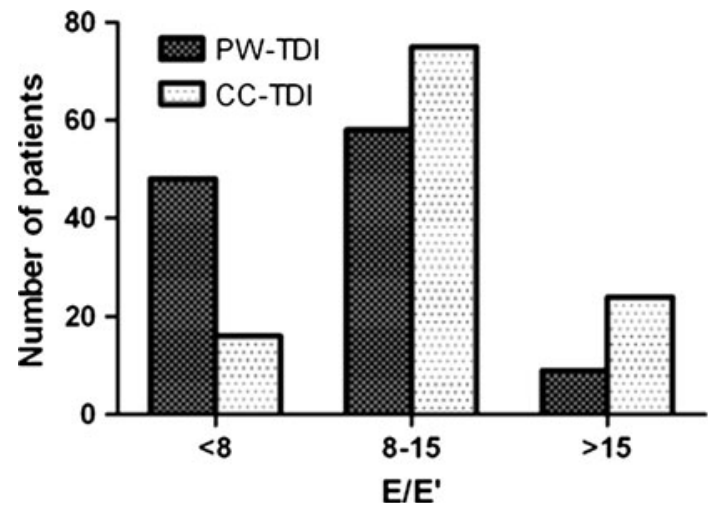

Fig. 5 Misclassification of diastolic dysfunction when PW-TDI cut-off values are used for CC-TDI

profile. However, the actual values that are obtained are markedly different. As a consequence, a significant difference in calculated $E / E^{\prime}$ values was found, which has important implications for the classification of patients with diastolic heart failure. Evaluation of diastolic function has become increasingly important, since it has been well established that nearly half of the patients that are hospitalized for acute decompensated heart failure have a relatively preserved systolic function, and diastolic dysfunction is considered to be the main cause of heart failure in these patients. Well-defined criteria for the diagnosis of diastolic heart failure are therefore important. A major step forward has been made with the consensus statement on the diagnosis of heart failure with normal left ventricular ejection fraction by the European Society of Cardiology [8]. One of the main measurements proposed to assess diastolic function is tissue velocity $E\left(E^{\prime}\right)$, and cut-off values have been agreed upon for classification of diastolic function using in particular the $E / E^{\prime}$ ratio. It is important to realize that the proposed $E / E^{\prime}$ cut-off values are based on pulsed Doppler measurements (PW-TDI). CC-TDI is routinely used in many clinics, and if they apply the same criteria for diastolic dysfunction as suggested in the ESC consensus statement, there will be a considerable overestimation of patients with diastolic dysfunction.

Previous studies comparing PW-TDI and CC-TDI have shown comparable results in phantoms, smaller cohorts of healthy volunteers and patients [14-16]. Kukulski et al. [11] investigated phantoms and 23 healthy volunteers (mean age 33 years), and found a good correlation between both methods but with unsatisfactory limits of agreement. McCulloch et al. [15] investigated 31 patients and showed significant correlations between myocardial velocities measured with PW-TDI and CC-TDI, with a significant underestimation of CC-TDI and consequently an overestimation of $E / E^{\prime}$. In their analysis of $E^{\prime}$ values, they found a relation which could be described by the following 
formula: $\quad E^{\prime} \quad(\mathrm{PW}-\mathrm{TDI})=1.46+1.14 \times E^{\prime} \quad(\mathrm{CC}-\mathrm{TDI})$. However, as they stated, a numeric uniform correction calculating PW-TDI-derived $E^{\prime}$ from CC-TDI-derived $E^{\prime}$ was hampered by the variable relationship ( $\sim 10-90 \%$ difference between values) as was shown in their Bland and Altmann analysis. Our Bland and Altmann analysis shows a better relationship ( $\sim 10-50 \%$ difference between values).

Tartiere et al. [16] compared PW-TDI and CC-TDI in 52 patients. They also found a similar difference between the two methods. Their Bland and Altman plot showed results comparable to our data for $E^{\prime}$ values between 3 and $11 \mathrm{~cm} / \mathrm{s}$. Values above $11 \mathrm{~cm} / \mathrm{s}$ in their group were sparse (four patients) compared to our population, and therefore our study gives more insight in the differences between both methods for a larger range of values. This leads to a more complete insight in the relation between PW-TDI and CC-TDI, showing increased differences with an increasing value of $E^{\prime}$.

The differences between the $E^{\prime}$ measurements can be partially explained by the different methodologies used for the estimation of myocardial velocities. PW-TDI is computed with a fast Fourier transformation (FFT) technique which will result in a peak velocity measured, while CC-TDI uses autocorrelation methodology resulting in peak-mean velocity. These limitations and differences in methodology were described in detail by Kukulski et al. [14]. A second explanation for the observed difference might be the placement of the sample within the myocardium. However, this explanation is less likely to account for the structural difference in TDI values since roughly the same myocardial region was sampled. Still slightly different transducer angulation and evaluation of different heart cycle may account for some of the differences. Other explanations might be related to the use of filters to reduce noise.

Technical disadvantages are more or less the same in both methodologies; however, CC-TDI has less spatial resolution and the advantage of offline measurements. Another advantage of CC-TDI methodology are other applications that can be performed on these tissue filtered images like tissue tracking and strain rate imaging.

\section{Limitations}

The applicability of our formula's to calculate $E^{\prime}$ and $E / E^{\prime}$ for PW-TDI using measurements of $E^{\prime}$ obtained with CCTDI or vice versa is not optimal since we also showed a large variability between values. The current formula, however, seems to be the best we have got so far. This variability can be partially explained by differences in methodology and slight differences in sample placing.
Also a slight difference in frame rates used in both methodologies of 180-211 fps might have increased this variability while due to 'undersampling' of CC-TDI peak velocities might have been missed.

\section{Conclusion}

In this study, we showed a strong correlation between $E^{\prime}$ values obtained by PW-TDI and CC-TDI. However, $E^{\prime}$ velocities obtained by CC-TDI are significantly lower. This may lead to different classification of diastolic function and erroneous diagnosis of diastolic heart failure in clinical practice using CC-TDI.

Open Access This article is distributed under the terms of the Creative Commons Attribution Noncommercial License which permits any noncommercial use, distribution, and reproduction in any medium, provided the original author(s) and source are credited.

\section{References}

1. Owan TE, Hodge DO, Herges RM, Jacobsen SJ, Roger VL, Redfield MM (2006) Trends in prevalence and outcome of heart failure with preserved ejection fraction. $\mathrm{N}$ Engl $\mathrm{J}$ Med 355(3):251-259

2. Abhayaratna WP, Marwick TH, Smith WT, Becker NG (2006) Characteristics of left ventricular diastolic dysfunction in the community: an echocardiographic survey. Heart 92(9):12591264

3. Voors AA, de Jong RM (2008) Treating diastolic heart failure. Heart 94(8):971-972

4. Massie BM, Carson PE, McMurray JJ, Komajda M, McKelvie R, Zile MR et al (2008) Irbesartan in patients with heart failure and preserved ejection fraction. N Engl J Med 359:2456-2467

5. Sanderson JE (2007) Heart failure with a normal ejection fraction. Heart 93(2):155-158

6. Ingle L, Cleland JG, Clark AL (2008) Perception of symptoms is out of proportion to cardiac pathology in patients with "diastolic heart failure". Heart 94(6):748-753

7. Hettwer S, Panzner-Grote B, Witthaut R, Werdan K (2007) Isolated diastolic dysfunction-diagnostic value of tissue Doppler imaging, colour M-mode and $\mathrm{N}$-terminal pro B-type natriuretic peptide. Clin Res Cardiol 96(12):874-882

8. Paulus WJ, Tschope C, Sanderson JE, Rusconi C, Flachskampf FA, Rademakers FE et al (2007) How to diagnose diastolic heart failure: a consensus statement on the diagnosis of heart failure with normal left ventricular ejection fraction by the Heart Failure and Echocardiography Associations of the European Society of Cardiology. Eur Heart J 28(20):2539-2550

9. Nagueh SF, Middleton KJ, Kopelen HA, Zoghbi WA, Quinones MA (1997) Doppler tissue imaging: a noninvasive technique for evaluation of left ventricular relaxation and estimation of filling pressures. J Am Coll Cardiol 30(6):1527-1533

10. Nagueh SF, Mikati I, Kopelen HA, Middleton KJ, Quinones MA, Zoghbi WA (1998) Doppler estimation of left ventricular filling pressure in sinus tachycardia. A new application of tissue Doppler imaging. Circulation 98(16):1644-1650

11. Ommen SR, Nishimura RA, Appleton CP, Miller FA, Oh JK, Redfield MM et al (2000) Clinical utility of Doppler 
echocardiography and tissue Doppler imaging in the estimation of left ventricular filling pressures: a comparative simultaneous Doppler-catheterization study. Circulation 102:1788-1794

12. Ommen SR, Nishimura RA (2003) A clinical approach to the assessment of left ventricular diastolic function by Doppler echocardiography: update 2003. Heart 89:iii18-iii23

13. Triantafyllou KA, Karabinos E, Kalkandi H, Kranidis AI, Babalis D (2009) Clinical implications of the echocardiographic assessment of left ventricular long axis function. Clin Res Cardiol 98:521-32

14. Kukulski T, Voigt JU, Wilkenshoff UM, Strotmann JM, Wranne B, Hatle L et al (2000) A comparison of regional myocardial velocity information derived by pulsed and color Doppler techniques: an in vitro and in vivo study. Echocardiography 17:639651
15. McCulloch M, Zoghbi WA, Davis R, Thomas C, Dokainish H (2006) Color tissue Doppler myocardial velocities consistently underestimate spectral tissue Doppler velocities: impact on calculation peak transmitral pulsed Doppler velocity/early diastolic tissue Doppler velocity (E/Ea). J Am Soc Echocardiogr 19:744748

16. Tartiere JM, Logeart D, Tartiere-Kesri L, Cohen-Solal A (2007) Color tissue Doppler underestimates myocardial velocity as compared to spectral tissue Doppler: poor reliability between both methods. Eur J Echocardiogr 9:268-272

17. Lang RM, Bierig M, Devereux RB, Flachskampf FA, Foster E, Pellikka PA et al (2006) Recommendations for chamber quantification. Eur J Echocardiogr 7:79-108 